\title{
Multiple genes are hypermethylated in intraductal papillary mucinous neoplasms of the pancreas
}

\author{
Seung-Mo Hong ${ }^{1}$, David Kelly ${ }^{1}$, Margaret Griffith ${ }^{1}$, Noriyuki Omura ${ }^{1}$, Ang Li ${ }^{1}$, \\ Chung-Pin $\mathrm{Li}^{2}$, Ralph H Hruban ${ }^{1,3}$ and Michael Goggins ${ }^{1,3,4}$
}

${ }^{1}$ Department of Pathology, The Sol Goldman Pancreatic Cancer Research Center, Johns Hopkins Medical Institutions, Johns Hopkins University, Baltimore, MD, USA; ${ }^{2}$ Division of Gastroenterology, Department of Medicine, Taipei Veterans General Hospital, National Yang-Ming University School of Medicine, Taipei, Taiwan; ${ }^{3}$ Department of Oncology, The Sol Goldman Pancreatic Cancer Research Center, Johns Hopkins Medical Institutions, Johns Hopkins University, Baltimore, MD, USA and ${ }^{4}$ Department of Medicine, The Sol Goldman Pancreatic Cancer Research Center, Johns Hopkins Medical Institutions, Johns Hopkins University, Baltimore, MD, USA

\begin{abstract}
Ductal adenocarcinoma of the pancreas is the fourth leading cause of cancer death and is usually diagnosed late. Intraductal papillary mucinous neoplasms are an increasingly recognized precursor to invasive ductal adenocarcinoma of the pancreas. Identifying the alterations in DNA methylation that arise during intraductal papillary mucinous neoplasm development may facilitate the development of markers that could be used to differentiate intraductal papillary mucinous neoplasms from non-neoplastic pancreatic cystic lesions. Surgically resected intraductal papillary mucinous neoplasms and adjacent ductal adenocarcinomas were microdissected from 50 patients. Normal pancreas was also obtained from 27 patients with intraductal papillary mucinous neoplasms or pancreatic adenocarcinomas and 10 patients with well-differentiated pancreatic endocrine neoplasms. Methylation-specific PCR was performed on isolated DNA for seven genes (SPARC, SARP2, TSLC1, RELN, TFPI2, CLDN5, UCHL1) known to be commonly aberrantly methylated in pancreatic ductal adenocarcinomas. The mean percentage of genes methylated in invasive ductal adenocarcinomas arising in association with an intraductal papillary mucinous neoplasm (mean \pm s.d., $81 \pm 17 \%$ ) was significantly higher than that in noninvasive-intraductal papillary mucinous neoplasms $(57 \pm 26 \%, P=0.007)$ or peritumoral normal epithelial cells $(22 \pm 17 \%, P<0.0001)$. Carcinomas (intraductal papillary mucinous neoplasms with carcinoma in situ or their associated infiltrating adenocarcinoma) had significantly more methylated genes $(71 \pm 19 \%)$ than low-grade (low and moderate dysplasia) intraductal papillary mucinous neoplasms (44 $\pm 26 \%$, $P<0.0001)$. The mean percentage of genes methylated in histologically normal pancreatic ductal cells from patients with ductal neoplasia $(22 \pm 17 \%)$ was significantly higher than in normal ductal cells from patients with well-differentiated pancreatic endocrine neoplasms $(4 \pm 7 \%, P=0.002)$. Thus, aberrant DNA methylation increases with histologic grades of intraductal papillary mucinous neoplasm. Low-level aberrant methylation in the normal ductal cells is more prevalent in patients with ductal neoplasia than in controls without ductal neoplasms and may contribute to carcinogenesis. The detection of aberrant methylation in pancreatic cystic lesions could facilitate the diagnosis of intraductal papillary mucinous neoplasms.
\end{abstract}

Modern Pathology (2008) 21, 1499-1507; doi:10.1038/modpathol.2008.157; published online 26 September 2008

Keywords: intraductal papillary mucinous neoplasm; DNA methylation; pancreatic cancer; SPARC; pancreatic endocrine neoplasm

Infiltrating ductal adenocarcinoma of the pancreas is the fourth leading cause of cancer death in United

Correspondence: Dr M Goggins, MD, Department of Pathology, Department of Medicine, Department of Oncology, The Sol Goldman Pancreatic Cancer Research Center, Johns Hopkins University, Johns Hopkins Medical Institutions, CRB2, Room 342, 1550 Orleans St, Baltimore, MD 21231, USA.

E-mail: mgoggins@jhmi.edu

Received 15 April 2008; revised 18 July 2008; accepted 19 July 2008; published online 26 September 2008
States and is usually diagnosed at a late stage. The detection and treatment of early stage pancreatic ductal adenocarcinomas and its precursors is perhaps the best way to reduce mortality from this disease. Intraductal papillary mucinous neoplasms (IPMNs) of the pancreas are an important precursor to infiltrating ductal adenocarcinoma of the pancreas. Unlike pancreatic intraepithelial neoplasms (PanINs) that are microscopic lesions of $<5 \mathrm{~mm}$, IPMNs are usually $\geq 1 \mathrm{~cm}$ in size and can be 
detected by imaging. ${ }^{1}$ They are also being increasingly identified as incidental lesions in patients who undergo abdominal imaging. ${ }^{2}$ The natural history of IPMNs is becoming better understood,,$^{1,3-5}$ with endosonographic and other features being used to predict their clinical behavior. ${ }^{6}$ Specifically, mainduct IPMNs are more likely to progress to invasive adenocarcinoma than branch-duct IPMNs, and branch-duct IPMNs have a low malignant potential until lesions are either $3 \mathrm{~cm}$ or more in size, are associated with dilatation of the main pancreatic duct, or are associated with symptoms such as abdominal pain. ${ }^{4,6,7}$ IPMNs can be cured if they are detected and resected before they progress to an invasive adenocarcinoma, ${ }^{5,8}$ although continued surveillance is necessary after resection as patients occasionally develop metachronous IPMNs in their remnant pancreas. ${ }^{9,10}$

The group that stands to benefit most from methods to detect asymptomatic IPMNs is patients with an inherited predisposition to pancreatic cancer (those with a family history in multiple family members or carriers of mutated familial pancreatic cancer susceptibility genes). ${ }^{11}$ Indeed, asymptomatic IPMNs have been detected and treated in individuals with a family history of pancreatic cancer who undergo screening. ${ }^{12,13}$

Molecular alterations that are thought to contribute to IPMNs have been identified. ${ }^{14}$ Known genetic alterations of IPMNs include mutations in the KRAS2 ${ }^{15,16}$ TP53, ${ }^{17}$ STK11/LKB1, ${ }^{18}$ and PIK3CA genes $^{19}$ as well as widespread chromosomal losses. ${ }^{20}$ Unlike PanINs, IPMNs rarely inactivate DPC4/ SMAD4. ${ }^{21,22}$ Recent studies have also identified numerous differentially expressed genes in IPMNs. $^{23,24}$ In principle, the detection of certain differentially expressed genes in pancreatic fluids could help in the diagnosis of these lesions, although few genes have been evaluated for this purpose..$^{25-27}$ Numerous genes have been identified that are aberrantly methylated and silenced in pancreatic ductal adenocarcinomas ${ }^{28-32}$ and several of these genes are known to undergo aberrant methylation in IPMNs including CDKN1C/ p57KIP2, ppENK, p16, Cyclin D2, SOCS-1, RELN, and TFPI2. ${ }^{29,31,33-35}$ These aberrantly methylated genes are promising targets for the early detection of pancreatic neoplasms. In this study, we evaluated the methylation status of seven genes (SPARC, SARP2, TSLC1, RELN, TFPI2, CLDN5, and UCHL1) in a large panel of IPMNs and as well as in peritumoral normal pancreatic ductal epithelia.

\section{Materials and methods}

\section{Patients and Tissues}

In total, 47 fresh-frozen IPMNs and 11 adjacent infiltrating ductal adenocarcinomas were collected from 50 patients with an IPMN who underwent pancreatic resection at the Johns Hopkins Medical
Institutions. Patient samples were selected according to the availability of stored tissues with sufficient numbers of neoplastic cells. Fresh-frozen normal pancreatic tissues from 27 patients with ductal neoplasms (3 with IPMNs and 24 with an infiltrating pancreatic ductal adenocarcinoma), and 10 patients with benign pancreatic endocrine neoplasms were used as controls. Hematoxylin and eosin staining of frozen sections was performed to assess the degree of dysplasia using criteria described elsewhere. ${ }^{36-38}$ The grade of dysplasia of IPMNs was classified into low grade, moderate, or high grade. ${ }^{39}$

In addition, formalin-fixed paraffin-embedded IPMN tissues were retrieved from 66 patients who underwent surgical resection at our institution and used to create tissue microarrays for immunohistochemical analyses. All specimens were collected and analyzed with the approval of the Johns Hopkins Committee for Clinical Investigation.

Normal pancreatic ductal epithelial cells were selectively microdissected from frozen sections adjacent to IPMN, pancreatic ductal adenocarcinoma, or benign endocrine neoplasms using the PALM micro laser system (Carl Zeiss microimaging Inc., North America, Thornwood, NY). Neoplastic cells from IPMNs were carefully collected by meticulous manual microdissection.

\section{Extraction and Bisulfite Treatment of DNA}

Genomic DNA was extracted from the microdissected samples with QIAamp DNA Micro Kit (Qiagen Inc., Valencia, CA, USA) following the manufacturer's protocol. Extracted DNA was quantified by either Quantifiler (Applied Biosystems, Foster City, CA, USA) or PicoGreen Assay (using a NanoDrop ND-3300 Fluorospectrometer, NanoDrop Technologies, Wilmington, DE, USA). Microdissected DNA was treated by sodium bisulfite (Sigma, St Louis, MO, USA) for $3 \mathrm{~h}$ at $70^{\circ} \mathrm{C}$ as previously described. ${ }^{40}$

\section{Methylation-Specific PCR}

The seven genes included in this study were selected because they have been previously observed to be frequently aberrantly methylated in primary pancreatic ductal adenocarcinomas and rarely methylated in normal pancreatic tissues. ${ }^{28-32}$ $S P A R C$ has been reported to be methylated in $88 \%$ of pancreatic ductal adenocarcinomas ${ }^{32}$ SARP2 in $95 \%,{ }^{28}$ TSLC1 in $27 \%,{ }^{30}$ RELN in $72 \%,{ }^{31}$ TFPI- 2 in $73 \%,{ }^{29}$ CLDN5 in $93 \%,{ }^{28}$ and UCHL1 in $100 \% .{ }^{28}$

The methylation status of $5^{\prime} \mathrm{CpG}$ islands of the seven target genes were detected by methylationspecific PCR (MSP) as previously described. ${ }^{40}$ After purification with the Wizard DNA clean-up system (Promega, Madison, WI, USA), $1 \mu \mathrm{l}$ of bisulfitetreated DNA was used for each $15 \mu$ l PCR reaction. 
The detection limit of each MSP assay was determined using the following concentrations of methylated DNA (1 ng, 500, 200, 100, 50, 25, 13, and $8 \mathrm{pg}$ and $0 \mathrm{pg}$ ). The lower limit of detection for methylated DNA was $25 \mathrm{pg}$ for RELN, TFPI2, and UCHL1, and $13 \mathrm{pg}$ for SPARC, TSLC1, SARP2, and CLDN5. As we suspected that any methylation present in normal pancreas tissues would be at lower concentrations than in IPMNs, we included more input DNA when amplifying DNA from normal pancreas than from the IPMNs. Thus, we generally used 710 ng DNA from manually microdissected normal pancreatic ductal epithelia, 0.4-2 ng for laser capture microdissected normal pancreatic duct DNA, and 0.05-2 ng DNA from manually dissected IPMNs (Figure 1). For seven IPMNs limited amounts of tissue were available, so only $40-100 \mathrm{pg}$ were used for MSP for these IPMNs. These concentrations of input DNA were well above the limit of detection of our MSP assays and there was no evidence of lower detectable methylation in these cases than in IPMNs with higher amounts of input DNA.

The primers used were designed previously to detect the sequence differences between methylated and unmethylated DNA as a result of bisulfite modification, and each primer pair contained at least four CpG sites to provide optimal specificity. Primer sequences for seven genes analyzed in the present study were described previously. ${ }^{28-32}$ PCR conditions were as follows: (1) $95^{\circ} \mathrm{C}$ for $5 \mathrm{~min}$; (2) 50 cycles of $95^{\circ} \mathrm{C}$ for $20 \mathrm{~s}, 60-62^{\circ} \mathrm{C}$ for $20 \mathrm{~s}$, and $72^{\circ} \mathrm{C}$ for $30 \mathrm{~s}$; and (3) final extension of $4 \mathrm{~min}$ at $72^{\circ} \mathrm{C}$. PCR products $(5 \mu \mathrm{l})$ were loaded onto $2.5 \%$ agarose gels and visualized by ethidium bromide staining.

\section{Immunohistochemistry}

Immunohistochemical analysis of SPARC expression was performed as previously described..$^{32,41}$ Briefly, tissue sections were deparaffinized and hydrated in xylene and serial alcohol solutions, respectively. Endogenous peroxidase was blocked by incubation in $3 \% \mathrm{H}_{2} \mathrm{O}_{2}$ for $10 \mathrm{~min}$. The antigen retrieval step was carried out in a steam pressure cooker containing preheated high $\mathrm{pH}$ antigen retrieval buffer (DakoCytomation, Glostrup, Denmark) at $95^{\circ} \mathrm{C}, 20 \mathrm{~min}$. To minimize nonspecific binding of antibodies, sections were incubated with a protein blocker (DakoCytomation) for $10 \mathrm{~min}$, and stained using the Envision Plus Detection method (DAKO, Carpinteria, CA, USA). Primary anti-SPARC monoclonal antibody (clone ON1-1, Zymed Laboratories Inc., San Francisco, CA, USA; 1:500 dilution) was applied manually to the sections and incubated for $60 \mathrm{~min}$ at room temperature. The remainder of the procedure was performed using an automated immunostainer (DakoCytomation). Immunostained sections were lightly counterstained in with hematoxylin, dehydrated in ethanol, and cleared in xylene. SPARC immunolabeling was categorized as absent when the labeling was absent to trace positive and the extent of labeling was less than $10 \%$ of cells. Immunolabeling was considered positive (expressed) if the intensity was moderate $(++)$, to strong $(+++)$, and the extent was more than or equal to $10 \%$ of cells. In most cases, the IPMNs that expressed SPARC showed diffuse labeling of the majority of neoplastic cells.

\section{Statistical Analysis}

The mean number of methylated genes was compared between IPMNs with and without associated invasive pancreatic ductal adenocarcinomas, between low-grade IPMNs (IPMN with low-grade dysplasia or IPMN with dysplasia) and high-grade IPMNs (IPMN with high-grade dysplasia and IPMNs with an associated infiltrating pancreatic ductal adenocarcinoma) and between IPMNs and normal pancreata. We also averaged the percentage of genes methylated out of the seven genes tested to get an overall percentage of genes methylated in an IPMN. ${ }^{28,42,43}$

Statistical analyses were performed using SPSS version 11 (SPSS Inc., Chicago, IL, USA). Comparison of means was performed using unpaired Student's t-test, Mann-Whitney, or one-way analysis of variance (ANOVA) tests. Pearson's correlations were performed to test the relationship between age and methylation. Linear regression analysis was performed to examine the relationship between patient age and the number of methylated genes. Associations between categorical variables were examined using the Pearson's $\chi^{2}$ - and Fisher's exact tests. A $P$-value of less than 0.05 was considered statistically significant.

\section{Results}

\section{Clinicopathologic Features of Cases}

The demographic profiles of the 50 patients whose IPMNs and associated infiltrating cancers were analyzed for DNA methylation are shown in Figure 1. Of which, 39 patients had IPMNs without an associated infiltrating adenocarcinoma and 11 patients had IPMNs with an associated invasive pancreatic ductal adenocarcinoma, all tubular type (colloid (mucinous) carcinomas were not included). Both the IPMN and the associated infiltrating cancer were available for analysis for 8 of the 11 patients (Figure 2). The 47 IPMNs included 12 with low-grade dysplasia (26\%), 12 with moderate dysplasia (26\%), and 23 with high-grade dysplasia (48\%). Twenty-eight cases $(59 \%)$ were classified as main-duct type, eleven (24\%) were branch-duct type, seven cases $(15 \%)$ were unknown, and one $(2 \%)$ was a combined main duct/branch-duct IPMN. Nineteen cases were pancreatobiliary $(40 \%)$, seventeen were gastric $(36 \%)$, and eleven were intestinal 

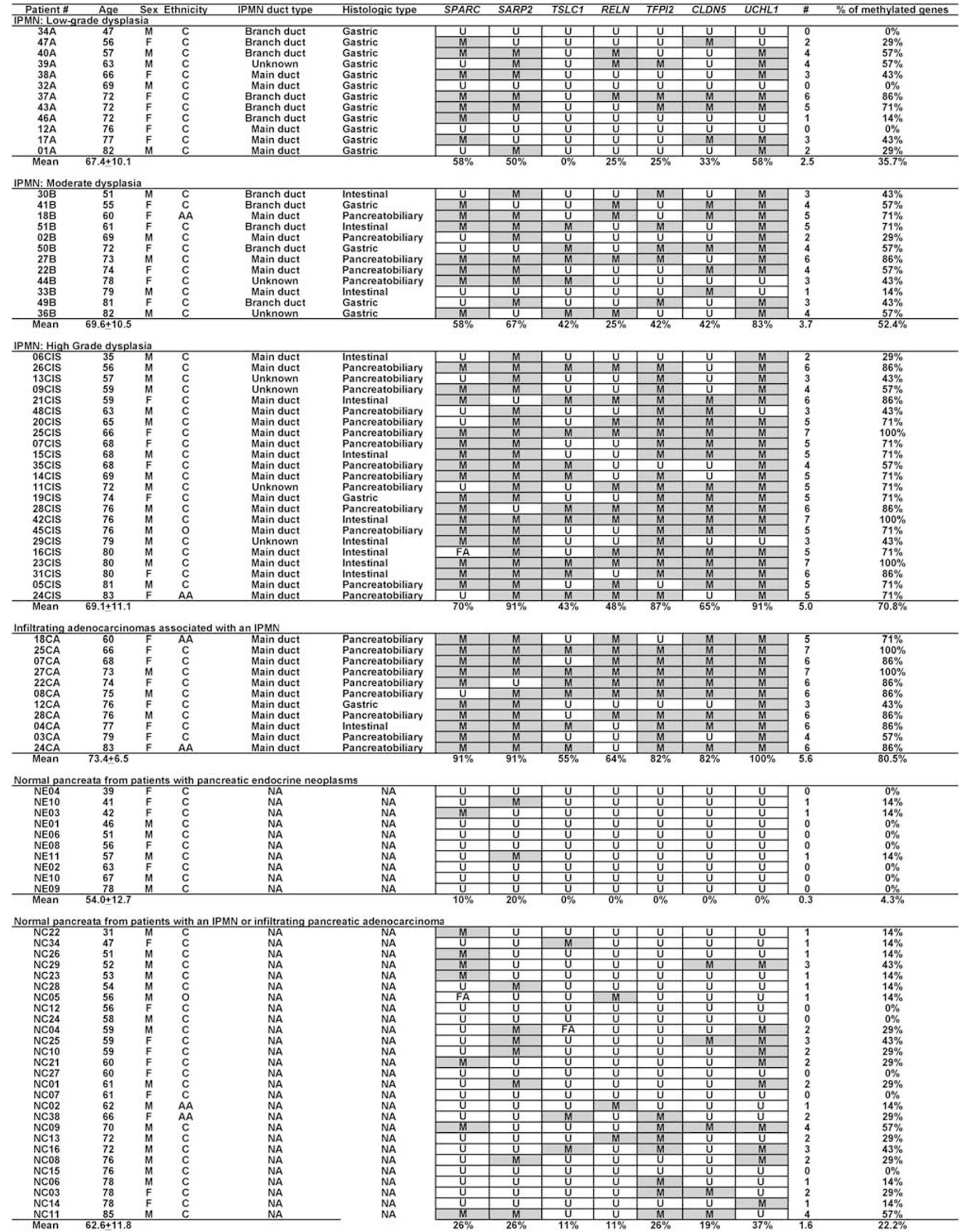

Figure 1 Summary of methylation profiles of IPMNs, infiltrating ductal adenocarcinomas arising in IPMNs, and peritumoral normal pancreatic tissues determined by MSP. Gray colored boxes, methylated alleles; open boxes, unmethylated alleles. M, methylated; U, unmethylated; FA, failed to amplify; NA, not applicable. 


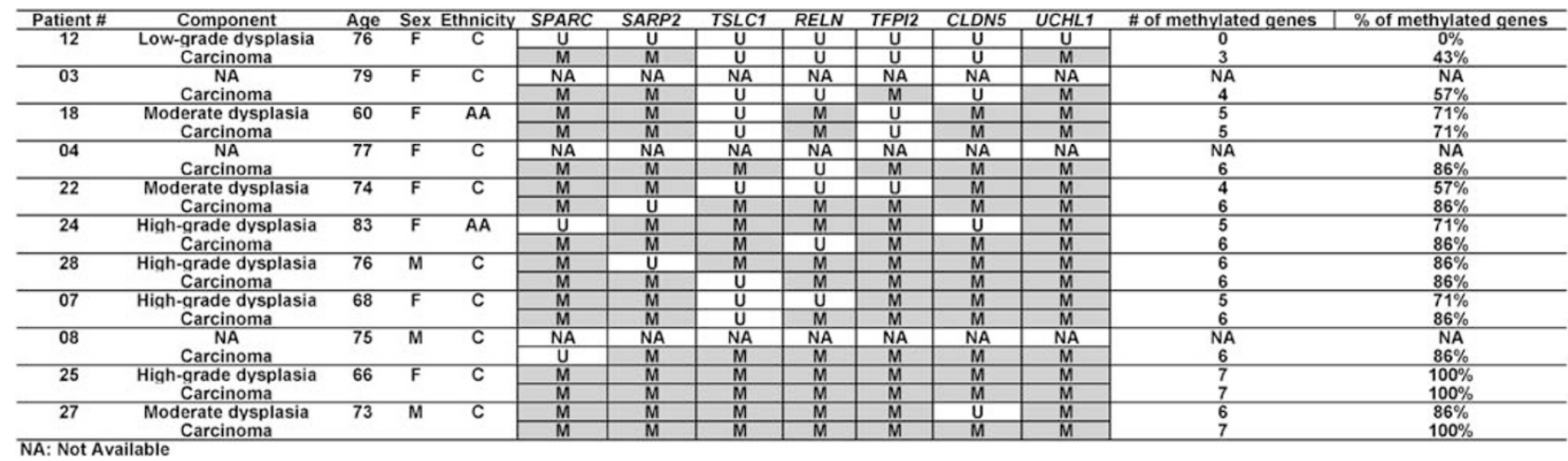

Figure 2 Methylation profiles of intraductal papillary mucinous neoplasms and their associated invasive adenocarcinomas. Gray colored boxes, methylated alleles; open boxes, unmethylated alleles.

(24\%) type by histologic type. All 12 gastric subtype IPMNs had low-grade dysplasia $(100 \%)$, whereas 8 of the 11 intestinal $(73 \%)$ and 14 of the 19 pancreatobiliary (74\%) IPMNs had high-grade dysplasia $(P<0.001)$. There was no significant difference between other demographic features and the histological subtypes.

\section{Methylation Analysis}

The DNA methylation profile of each IPMN and the normal pancreatic tissues is shown in Figure 1. Overall, the mean percentage of genes methylated in the infiltrating pancreatic ductal adenocarcinomas arising in association with an IPMN ( 7 genes $\times 11$ samples $=77$ possible genes per samples) was $81 \pm 17 \%$ (62 methylated genes per samples) higher than in normal pancreas samples $(22 \pm 17 \% ; 42$ methylated genes of a possible 189 genes per samples, $P<0.0001)$. The percentage of genes methylated in the infiltrating pancreatic ductal adenocarcinomas associated with an IPMN was also higher than in the IPMNs without accompanying invasive cancer $(57 \pm 26 \%$; $t$-test, $P=0.007)$. The percentage of genes methylated in IPMNs $(66 \pm 26 \%)$ was higher than in normal pancreas $(P<0.0001)$. The mean percentage of methylated genes was also compared according to grade of IPMN; IPMNs with low-grade dysplasia (mean, 36 $\pm 29 \%$ ), moderate dysplasia $(52 \pm 20 \%)$, and high-grade dysplasia $(71 \pm 19 \%)$. The mean percentage of methylated genes differed significantly among IPMN grades (one-way ANOVA, $P<0.0001$ ). We then compared the percentage of methylated genes within each grade of IPMN. The mean percentage of methylated genes was higher in IPMNs with high-grade dysplasia than in IPMNs with moderate dysplasia (MannWhitney $U$-test, $P<0.0001)$. However, there was no significant difference in the number of methylated genes in IPMNs with low-grade dysplasia vs IPMNs with moderate dysplasia (data not shown). Similarly, low-grade IPMNs (IPMNs with low-grade dysplasia and IPMNs with moderate dysplasia, $n=24)$ showed significantly less methylation $(44 \pm 26 \%)$ than high-grade IPMNs (IPMNs with high-grade dysplasia and invasive adenocarcinomas; $n=23,71 \pm 19 \%, P<0.0001)$. However, there was no significant difference in the prevalence of methylation between IPMNs with an invasive adenocarcinoma ( $n=8,66 \pm 30 \%)$ and those without invasive adenocarcinomas $(n=39, \quad 55 \% \pm 25 \%$, Mann-Whitney $U$-test), although this comparison may be underpowered. In addition, there was no difference in the mean number of methylated genes between main-duct $(4 \pm 2)$ and branch-duct $(3 \pm 2)$ IPMNs. The prevalence of aberrant methylation was also compared for each histologic subtype of IPMN; the mean percentage of genes methylated in gastrictype IPMNs was $42 \pm 29 \%$, intestinal-type $64 \pm 29 \%$, and pancreatobiliary-type $66 \pm 18 \%$. Although the mean percentage of methylated genes differed significantly among histologic types of IPMN (oneway ANOVA, $P=0.009$ ) and was lower in gastric than in intestinal or pancreaticobiliary, all gastrictype IPMNs were low-grade adenomas, precluding any comparisons of histologic type independent of stage.

We next examined if there were any differences in the prevalence of methylated genes in normal pancreatic tissues by age, gender, or disease. Methylation patterns did not differ by gender. The mean percentage of methylated genes in normal pancreatic ductal cells from patients with ductal neoplasia $(22 \pm 17 \%)$ was significantly higher than in normal ductal cells from patients with welldifferentiated pancreatic endocrine neoplasms (4 $\pm 7 \%$, Mann-Whitney $U$-test, $P=0.002$ ). The mean age of the patients that underwent analysis of their normal pancreas ( $63 \pm 12$ years) was marginally higher in those with ductal neoplasia than in those with well-differentiated pancreatic endocrine neoplasms $(54 \pm 13$ years, $P=0.06)$. When we matched these two groups by age comparing methylation of normal pancreata in younger members of the ductal neoplasia group (patients with an 
IPMNs or infiltrating ductal adenocarcinoma, and an age $<70$, mean age $56 \pm 7$ ) to that of the endocrine neoplasm group, there was still a significantly more methylation in the ductal neoplasia group $(1 \pm 1$ genes) than in the endocrine neoplasm group $(0.3 \pm 0.5$ genes, $P=0.002)$. Within the pancreatic ductal adenocarcinoma group, there was a modest correlation between patient age and the prevalence of methylation in their normal pancreas $(R=0.34$, $P=0.08)$.

We examined the progression of individual gene methylation with neoplastic grade. We determined if there was any significant difference in the prevalence of methylation individual genes by neoplastic grade (low-grade dysplasia, moderate dysplasia, high-grade dysplasia, and infiltrating pancreatic ductal adenocarcinoma). There was a significant difference in prevalence in the methylation of SARP2 $\left(P=0.02, \chi^{2}\right.$-test $), T S L C 1(P=0.03)$, TFPI2 $(P=0.001)$, UCHL1 $(P=0.03)$, and CLDN5 $(P=0.06)$ by neoplastic grade, but not of methylation SPARC $(P=0.47)$ or RELN $(P=0.24)$. We then analyzed methylation prevalence by grade. Low-grade IPMNs (low-grade dysplasia, moderate dysplasia $)(n=24)$ were compared to advanced neoplasms (IPMNs with high-grade dysplasia and IPMNs associated with an invasive adenocarcinoma; $n=34)$. Aberrant methylation of $S A R P 2(P=0.004)$, TSLC1 $\quad(P=0.04), \quad$ TFPI2 $\quad(P<0.0001), \quad C L D N 5$ $(P=0.01)$, and $U C H L 1(P=0.02)$ were detected more often in the more advanced lesions than in the lowgrade IPMNs (Figure 1). Within the IPMNs, aberrant methylation of SARP2 $(P=0.01)$ and TFPI2 $(P<0.0001)$ were detected more often in high-grade IPMNs (IPMNs with high-grade dysplasia, $n=23$ ) than in those with low-grade IPMNs (low-grade dysplasia, moderate dysplasia; Figure 1) and aberrant methylation of TSLC1 $(P<0.0001)$ was detected more often in high-grade IPMNs (IPMNs with highgrade dysplasia) than in IPMNs with low-grade dysplasia.

\section{SPARC Expression Analysis}

We also determined if aberrant methylation in IPMNs was associated with gene silencing. We examined expression of SPARC by immunohistochemistry in 66 IPMNs (Table 1 and Figure 3). SPARC expression was observed in $50 \%$ of IPMNs with low-grade dysplasia (4 of 8) and IPMNs with moderate dysplasia (12 of 24 ), but in only $20 \%$ of IPMNs with high-grade dysplasia (7 of $34 ; \chi^{2}$-test, $P=0.04)$. Loss of SPARC expression was not associated with clinicopathologic factors such as histologic subtype or IPMN location.

\section{Discussion}

In this study, we demonstrate that aberrant DNA methylation is a common feature of IPMNs and
Table 1 SPARC protein expression of IPMNs

\begin{tabular}{|c|c|c|c|c|}
\hline & $\begin{array}{l}\text { SPARC expression } \\
\text { Minimal or absent }\end{array}$ & Present & Total & $\mathrm{P}$-value \\
\hline \multicolumn{5}{|l|}{ Histologic grade } \\
\hline Low grade & 4 & 4 & 8 & $0.04^{*}$ \\
\hline Moderate & 12 & 12 & 24 & \\
\hline High grade & 27 & 7 & 34 & \\
\hline \multicolumn{5}{|l|}{ Histologic subtype } \\
\hline Pancreatobiliary & 22 & 14 & 36 & 0.86 \\
\hline Intestinal & 14 & 6 & 20 & \\
\hline Gastric & 5 & 2 & 7 & \\
\hline Oncocytic & 1 & 0 & 1 & \\
\hline Unclassified & 1 & 1 & 2 & \\
\hline \multicolumn{5}{|l|}{ Location } \\
\hline Main duct & 27 & 10 & 37 & 0.36 \\
\hline Branch duct & 9 & 9 & 18 & \\
\hline Combined & 4 & 3 & 7 & \\
\hline Unknown & 3 & 1 & 4 & \\
\hline
\end{tabular}

* Significant at the level of $P<0.05$ (adenoma+borderline vs CIS).

increases with histologic grade. We find that overall prevalence of methylation in IPMNs with high-grade dysplasia is similar to that observed for associated pancreatic ductal adenocarcinomas. We also find that the prevalence of methylation in IPMNs with high-grade dysplasia was significantly higher than that in IPMNs with moderate dysplasia. Overall, these results indicate that for our gene panel aberrant methylation increases in prevalence through increasing histologic grades and is largely complete prior to the onset of invasive adenocarcinoma.

We also find that patients with ductal neoplasia have more low-level aberrant methylation of our gene panel in their normal pancreatic ductal epithelial cells than do patients with pancreatic endocrine neoplasms. This finding was observed even when we controlled for age. These results suggest that the propensity to develop aberrant methylation in histologically normal tissues is not merely a function of age and may predispose patients to the development of ductal neoplasia. These results expand previous findings in which we demonstrated patients with pancreatic adenocarcinoma have a higher prevalence of methylated DNA in their normal duodenum than patients with pancreatitis. ${ }^{42}$ Similarly, studies in other organ systems indicate that adjacent mucosa from patients with adenocarcinoma contain more aberrant methylation than matched controls. In principle, the detection of such low-level methylation in histologically normal tissues could be used to stratify an individual's cancer risk but large long-term prospective studies would be required using an as-yet-to-bedefined optimal marker panel to test this hypothesis.

We also demonstrate that IPMNs show frequent loss of expression of the protein product of SPARC, and that this loss is more common in high-grade IPMNs than in lower grade IPMNs. We have 

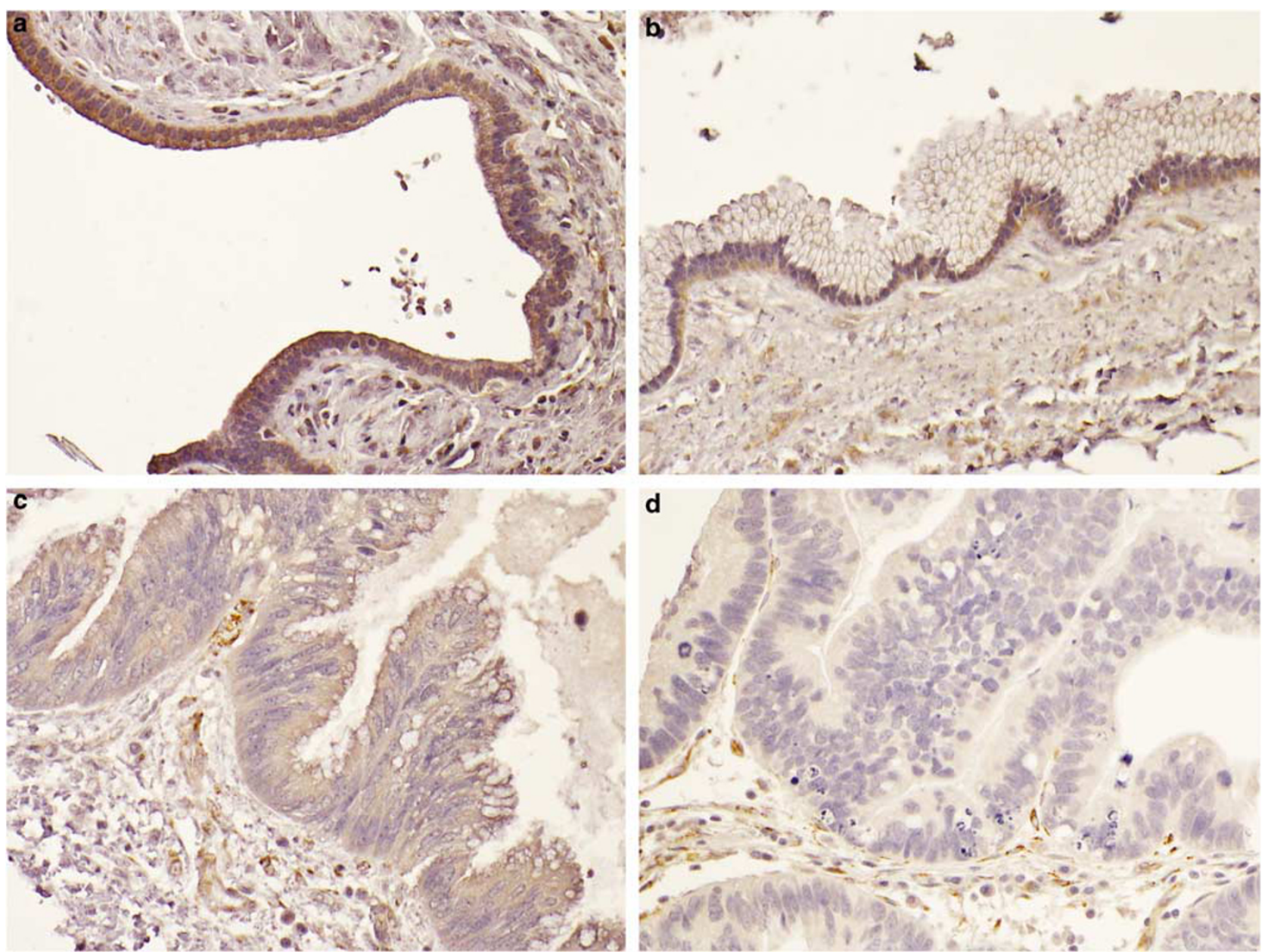

Figure 3 Sparc protein expression in normal pancreatic ductal epithelial cells and IPMNs. (a) Sparc labeling in normal pancreatic ductal epithelial cells $(\times 20)$. (b) An IPMN with low-grade dysplasia shows strong Sparc positivity in tumor cells $(\times 20)$. $(\mathbf{c})$ An IPMN with moderate dysplasia shows weak sparc positivity in tumor cells $(\times 20)$. (d) An IPMN with high-grade dysplasia demonstrated loss of Sparc labeling $(\times 20)$. Occasional fibroblasts in stroma in each figure show strong Sparc expression are used as internal positive controls.

previously demonstrated that aberrant methylation of SPARC in pancreatic cancers is associated with gene silencing ${ }^{32}$ and interference with tumor stromal interactions. ${ }^{32,41}$ The prevalence of SPARC methylation in IPMNs with moderate or high-grade dysplasia was similar to the prevalence in infiltrating pancreatic ductal adenocarcinomas $(88 \%){ }^{32}$

The aberrant methylation of the other genes in our panel may have functional consequences in the progression of IPMNs. For example, TSLC1/IGSF1 encodes a protein with structural homology to cell adhesion molecules and may be involved in intercellular interactions. ${ }^{30}$ Methylation of TSLC1 was only observed in IPMNs with moderate or higher grade dysplasia suggesting its detection in pancreatic cystic lesions could be useful for predicting a more advanced grade of IPMN. This finding mirrors our previous results of methylation profiles of PanINs, in which methylation was observed only from PanIN-3 lesions. ${ }^{30}$ TFPI2 (tissue factor pathway inhibitor 2) encodes a serine proteinase inhibitor that is frequently inactivated by aberrant methyla- tion in pancreatic adenocarcinomas. ${ }^{29}$ Reelin, the protein product of RELN is a key regulator of neuronal migration, is also commonly silenced in pancreatic ductal adenocarcinomas and epigenetic treatment of pancreatic cancer cells restores RELN expression and significantly inhibits tumor cell migration. We have previously demonstrated that IPMNs often show loss of expression of the protein product of RELN. The protein product of CLDN5 (claudin 5) is a component of tight junctions, and was methylated in $81 \%$ of pancreatic ductal adenocarcinomas (35 of 43) and in 42\% (10 of 24) of juice samples from patients with pancreatic ductal adenocarcinomas. SARP2/SFRP1 (secreted apoptosisrelated protein 2) is a member of SARP genes family that counteracts the Wnt oncogenic signaling pathway and is considered to be involved in apoptosis. UCHL1/PGP9.5 is known to be methylated in several other cancers, including esophageal, stomach, and head and neck cancers.

Non-neoplastic cysts of the pancreas are relatively common, and it can be difficult to distinguish a 
neoplastic cyst that may progress to invasive cancer from a non-neoplastic cyst that does not require treatment. Our results indicate that a DNA methylation panel may be helpful in facilitating the detection of IPMNs and differentiating neoplastic IPMNs from non-neoplastic cystic lesions. We have previously demonstrated that pancreatic fluids such as pancreatic juice could be used as a source of molecular markers of pancreatic neoplasia. ${ }^{43,44}$ Quantitative methylation analysis of pancreatic juice can distinguish patients with pancreatic cancer from patients with non-neoplastic pancreatic disease with high accuracy. A panel of DNA methylation and other molecular markers are being evaluated for their utility to detect neoplasia in patients undergoing screening because of a strong family history of pancreatic cancer. ${ }^{11,43,45}$

Ultimately, it may be possible to infer the likely histological grade or risk of progression of a neoplastic cystic lesion based on a panel of molecular markers. In such a scenario, the analysis of fine needle aspirates to detect a threshold concentration of accurate aberrant DNA methylation or other markers whose behavior in different grades of resected IPMNs had been extensively characterized, could be useful in predicting their histological grade prior to resection. Such an application would require a panel of markers with high specificity for each grade, those with characteristics such as methylated TSLC1 that was predictive of having an IPMN of moderate grade or higher.

In summary, we find aberrant methylation of a seven-gene panel is a common feature of IPMNs and increases with histologic grade. The detection of aberrant methylated genes may help in the early detection and diagnostic evaluation of cystic lesions of the pancreas. Aberrant methylation in the normal pancreas is detected more often in patients with ductal neoplasia than in controls without ductal neoplasms and may therefore to contribute to carcinogenesis.

\section{References}

1 Hruban RH, Takaori K, Klimstra DS, et al. An illustrated consensus on the classification of pancreatic intraepithelial neoplasia and intraductal papillary mucinous neoplasms. Am J Surg Pathol 2004;28:977-987.

2 Winter JM, Cameron JL, Lillemoe KD, et al. Periampullary and pancreatic incidentaloma: a single institution's experience with an increasingly common diagnosis. Ann Surg 2006;243:673-680; discussion 80-83.

3 D'Angelica M, Brennan MF, Suriawinata AA, et al. Intraductal papillary mucinous neoplasms of the pancreas: an analysis of clinicopathologic features and outcome. Ann Surg 2004;239:400-408.

4 Salvia R, Fernandez-del Castillo C, Bassi C, et al. Mainduct intraductal papillary mucinous neoplasms of the pancreas: clinical predictors of malignancy and longterm survival following resection. Ann Surg 2004;239:678-685; discussion 85-87.
5 Chari ST, Yadav D, Smyrk TC, et al. Study of recurrence after surgical resection of intraductal papillary mucinous neoplasm of the pancreas. Gastroenterology 2002;123:1500-1507.

6 Kobayashi G, Fujita N, Noda Y, et al. Mode of progression of intraductal papillary-mucinous tumor of the pancreas: analysis of patients with follow-up by EUS. J Gastroenterol 2005;40:744-751.

7 Salvia R, Fernandez-del Castillo C, Bassi C, et al. Mainduct intraductal papillary mucinous neoplasms of the pancreas: clinical predictors of malignancy and longterm survival following resection. Ann Surg 2004;239:678-685; discussion 85-87.

8 Sohn TA, Yeo CJ, Cameron JL, et al. Intraductal papillary mucinous neoplasms of the pancreas: an updated experience. Ann Surg 2004;239:788-797; discussion 97-99.

9 Bassi C, Sarr MG, Lillemoe KD, et al. Natural history of intraductal papillary mucinous neoplasms (IPMN): current evidence and implications for management. J Gastrointest Surg 2008;12:645-650.

10 White R, D'Angelica M, Katabi N, et al. Fate of the remnant pancreas after resection of noninvasive intraductal papillary mucinous neoplasm. J Am Coll Surg 2007;204:987-993; discussion 93-95.

11 Canto MI, Goggins M, Hruban RH, et al. Screening for early pancreatic neoplasia in high-risk individuals: a prospective controlled study. Clin Gastroenterol Hepatol 2006;4:766-781; quiz 665.

12 Klein AP, Brune KA, Petersen GM, et al. Prospective risk of pancreatic cancer in familial pancreatic cancer kindreds. Cancer Res 2004;64:2634-2638.

13 Brune K, Goggins M, O’Mailey L, et al. Detailed pathologic evaluation of non-invasive precursor lesions of the pancreas in patients with a strong family history of pancreatic cancer. Am J Surg Pathol 2006;30:1067-1076.

14 Hruban RH, Maitra A, Kern SE, et al. Precursors to pancreatic cancer. Gastroenterol Clin North Am 2007;36:831-849.

15 Z'Graggen K, Rivera JA, Compton CC, et al. Prevalence of activating K-ras mutations in the evolutionary stages of neoplasia in intraductal papillary mucinous tumors of the pancreas. Ann Surg 1997;226:491-498.

16 Schonleben F, Qiu W, Bruckman KC, et al. BRAF and KRAS gene mutations in intraductal papillary mucinous neoplasm/carcinoma (IPMN/IPMC) of the pancreas. Cancer Lett 2007;249:242-248.

17 Sessa F, Solcia E, Capella C, et al. Intraductal papillary-mucinous tumours represent a distinct group of pancreatic neoplasms: an investigation of tumour cell differentiation and K-ras, p53 and c-erbB-2 abnormalities in 26 patients. Virchows Arch 1994;425:357-367.

18 Sato N, Rosty C, Jansen M, et al. STK11/LKB1 PeutzJeghers gene inactivation in intraductal papillarymucinous neoplasms of the pancreas. Am J Pathol 2001;159:2017-2022.

19 Schonleben F, Qiu W, Ciau NT, et al. PIK3CA mutations in intraductal papillary mucinous neoplasm/carcinoma of the pancreas. Clin Cancer Res 2006;12:3851-3855.

20 Abe T, Fukushima N, Brune K, et al. Genome wide allelotypes of familial pancreatic adenocarcinomas and familial and sporadic intraductal papillary mucinous neoplasms. Clin Cancer Res 2007;13: 6019-6025. 
21 Iacobuzio-Donahue CA, Klimstra DS, Adsay NV, et al. Dpc-4 protein is expressed in virtually all human intraductal papillary mucinous neoplasms of the pancreas: comparison with conventional ductal adenocarcinomas. Am J Pathol 2000;157:755-761.

22 Wilentz RE, Iacobuzio-Donahue CA, Argani P, et al. Loss of expression of Dpc4 in pancreatic intraepithelial neoplasia: evidence that DPC4 inactivation occurs late in neoplastic progression. Cancer Res 2000;60:2002-2006.

23 Terris B, Blaveri E, Crnogorac-Jurcevic $\mathrm{T}$, et al. Characterization of gene expression profiles in intraductal papillary-mucinous tumors of the pancreas. Am J Pathol 2002;160:1745-1754.

24 Sato N, Fukushima N, Maitra A, et al. Gene expression profiling identifies genes associated with invasive intraductal papillary mucinous neoplasms of the pancreas. Am J Pathol 2004;164:903-914.

25 Ohuchida K, Mizumoto K, Fujita H, et al. Sonic hedgehog is an early developmental marker of intraductal papillary mucinous neoplasms: clinical implications of mRNA levels in pancreatic juice. J Pathol 2006;210:42-48.

26 Schmidt CM, Yip-Schneider MT, Ralstin MC, et al. PGE(2) in pancreatic cyst fluid helps differentiate IPMN from MCN and predict IPMN dysplasia. J Gastrointest Surg 2008;12:243-249.

27 Koopmann J, Buckhaults P, Brown DA, et al. Serum macrophage inhibitory cytokine 1 as a marker of pancreatic and other periampullary cancers. Clin Cancer Res 2004;10:2386-2392.

28 Sato N, Fukushima N, Maitra A, et al. Discovery of novel targets for aberrant methylation in pancreatic carcinoma using high-throughput microarrays. Cancer Res 2003;63:3735-3742.

29 Sato N, Parker AR, Fukushima N, et al. Epigenetic inactivation of TFPI-2 as a common mechanism associated with growth and invasion of pancreatic ductal adenocarcinoma. Oncogene 2005;24:850-858.

30 Jansen M, Fukushima N, Rosty C, et al. Aberrant methylation of the $5^{\prime} \mathrm{CpG}$ island of TSLC1 is common in pancreatic ductal adenocarcinoma and is first manifest in high-grade PanlNs. Cancer Biol Ther 2002;1:293-296.

31 Sato N, Fukushima N, Chang R, et al. Differential and epigenetic gene expression profiling identifies frequent disruption of the RELN pathway in pancreatic cancers. Gastroenterology 2006;130:548-565.

32 Sato N, Fukushima N, Maehara N, et al. SPARC/ osteonectin is a frequent target for aberrant methylation in pancreatic adenocarcinoma and a mediator of tumor-stromal interactions. Oncogene 2003;22: 5021-5030.
33 Sato N, Rosty C, Jansen M, et al. STK11/LKB1 PeutzJeghers gene inactivation in intraductal papillarymucinous neoplasms of the pancreas. Am J Pathol 2001;159:2017-2022.

34 House MG, Guo M, Iacobuzio-Donahue C, et al. Molecular progression of promoter methylation in intraductal papillary mucinous neoplasms (IPMN) of the pancreas. Carcinogenesis 2003;24:193-198.

35 Sato N, Ueki T, Fukushima N, et al. Aberrant methylation of $\mathrm{CpG}$ islands in intraductal papillary mucinous neoplasms of the pancreas. Gastroenterology 2002;123:365-372.

36 Longnecker DS, Adsay NV, Fernandez-del Castillo C, et al. Histopathological diagnosis of pancreatic intraepithelial neoplasia and intraductal papillary-mucinous neoplasms: interobserver agreement. Pancreas 2005;31:344-349.

37 Furukawa T, Kloppel G, Volkan Adsay N, et al. Classification of types of intraductal papillary-mucinous neoplasm of the pancreas: a consensus study. Virchows Arch 2005;447:794-799.

38 Andrejevic-Blant S, Kosmahl M, Sipos B, et al. Pancreatic intraductal papillary-mucinous neoplasms: a new and evolving entity. Virchows Arch 2007;451:863-869.

39 Hruban RH, Pitman MB, Klimstra DS. Tumors of the Pancreas. Atlas of Tumor Pathology. American Registry of Pathology and Armed Forces Institute of Pathology: Washington, DC, 2007.

40 Herman JG, Graff JR, Myohanen S, et al. Methylationspecific PCR: a novel PCR assay for methylation status of CpG islands. Proc Natl Acad Sci USA 1996;93: 9821-9826.

41 Infante JR, Matsubayashi H, Sato N, et al. Peritumoral fibroblast SPARC expression and patient outcome with resectable pancreatic adenocarcinoma. J Clin Oncol 2007;25:319-325.

42 Matsubayashi $\mathrm{H}$, Sato N, Brune K, et al. Age- and disease-related methylation of multiple genes in nonneoplastic duodenum and in duodenal juice. Clin Cancer Res 2005;11:573-583.

43 Matsubayashi H, Canto $\mathrm{M}$, Sato $\mathrm{N}$, et al. DNA methylation alterations in the pancreatic juice of patients with suspected pancreatic disease. Cancer Res 2006;66:1208-1217.

44 Fukushima N, Walter KM, Uek T, et al. Diagnosing pancreatic cancer using methylation specific PCR analysis of pancreatic juice. Cancer Biol Ther 2003:2:78-83.

45 Canto MI, Goggins M, Yeo CJ, et al. Screening for pancreatic neoplasia in high-risk individuals: an EUSbased approach. Clin Gastroenterol Hepatol 2004;2:606-621. 